\title{
The Status of the FAST Project
}

\section{Bo Peng}

National Astronomical Observatories, Chinese Academy of Sciences

Datun Rd. A20, Chaoyang District, Beijing 100012, China

E-mail: pbenao.cas.cn

With a huge collecting area of more than 30 football fields, the FAST (Five-hundred-meter Aperture Spherical Telescope) will become the largest single dish ever built in the world. The construction of the project FAST will be started officially in October 2010. The current status of the project is updated in this paper, focussed on FAST site preparation, progress on key system designs of the main active reflector, feed driving and pointing, measurements and controls, feeds and receivers, etc. Finally we introduce the newly established JLRAT (Joint Laboratory for Radio Astronomy Technology), to enhance Chinese involvements in the international SKA (Square Kilometre Array) project.

ISKAF2010 Science Meeting - ISKAF2010

Assen, the Netherlands

June 10-14 201 


\section{Introduction}

The SKA (Square Kilometre Array) has been grown up as a global collaboration project since it was born in early 1990s. There are now about 20 countries involved in the SKA project in different ways. The FAST (Five-hundred-meter Aperture Spherical Telescope), which can be seen as a forerunner of the SKA, is a funded Chinese National Large Scale Facility project. It will be constructed in about 5.5 years from now.

We will introduce the long path to the FAST which was rooted from the SKA, bring up recent updates on FAST project, and make a plan for future Chinese participation to the SKA, which is coordinated by the JLRAT (Joint Laboratory for Radio Astronomy Technology).

\subsection{Chinese concept for the SKA}

After some independent suggestions between a few individual astronomers around early 1990s, radio astronomers from 10 countries including China, meeting at the 24th General Assembly of the URSI (International Union for Radio Science) in Kyoto Japan in 1993, proposed to build a new generation large radio telescope (called LT, renamed to the SKA in 1999), with an effective collecting area of about one square kilometers. The LTWG (Large Telescope Working Group) was then formed to coordinate international efforts as a start point. In 1994 China set up a LT resaerch group at the BAO (Beijing Astronomical Observatory, Chinese Academy of Sciences), working at realizing the SKA with a large spherical reflector array, i.e., a Chinese SKA concept KARST (Kilometer Area Radio Synthesis Telescope) was born, by making use of the karst landforms existed extensively in Guizhou province of China. The KARST concept is a representative of the so-called LDSN (large diameter small number) concept for building up the SKA (Nan et al., 2002). Astronomers and engineerings have been worked at RFI (radio frequency interference) quiet site survey in the karst area at Pintang and Puding counties in Guizhou province, light focus positioning and wideband feed studies among 15 Chinese institutes and universities since then. Early results (Strom et al., 1996) of which were reported at the third Large Telescope Working Group and of a workshop on spherical telescopes conference (LTWG-3) held in Guizhou province in October 1995.

\subsection{FAST project}

The KARST concept (Peng et al., 1998, Nan et al., 2002) became mature mostly by the progress on three key technical issues, karst sinkhole as a site, feed positioning by cable driven, and the giant reflector shaped by actuators. Around the summer 1997, the FAST was proposed to be an innovative concept between a fully steerable dish like Effelsberg $100 \mathrm{~m}$ radio telescope in Germany, and a fixed dish like Arecibo $305 \mathrm{~m}$ radio telescope in Puerto Rico. In a word, FAST is a modified "Arecibo" type radio telescope with its big reflector adjustable to conform a parabolic revolution from a sphere shape, while the focus cabin is driven and pointed by suspension long cables simultaneously, integrating optical, mechanical and electronic technologies. The FAST can be seen as a prototype of the KARST, a Chinese SKA concept. 
Preparatory study on the FAST project was first funded by the CAS (Chinese Academy of Sciences) together with the MOST (Ministry of Science and Technology, China) in 1998, to study and prototype key technical issues intensively. Continued supports were received from the CAS and NSFC (National natural Science Foundation of China) for FAST's feasibility studies and preliminary designs. To assemble all key technologies to be applied to the FAST construction, Miyun FAST demonstrator, namely MyFAST, was constructed successfully by September 2006 by detecting neutral hydrogen in the Milky Way.

In July 2007, FAST was approved as a National Large Scale Scientific Facility by the NDRC (National Development and Reform Commission). In 2009 the construct funds started to flow, the official opening of the FAST construction is scheduled around October 2010, and the FAST commissioning is expected to be in late 2015.

\section{Critical technology developments for FAST project}

The optical geometry of the FAST is shown in Figure 1 (left panel), where the reflector is a spherical cap with a radius of $\mathrm{R} \sim 300 \mathrm{~m}$ and an opening aperture of $500 \mathrm{~m}$ in diameter. The effective aperture of $\sim 300 \mathrm{~m}$ will be illuminated by the feeds in a cabin moving on the focus surface, halfway from the reflector to its spherical center. The receivers will cover a frequency range from $70 \mathrm{MHz}$ to $3 \mathrm{GHz}$, with extendable capability up to $8 \mathrm{GHz}$ for future. Upgrade. The telescope is to be pointed by moving the focus cabin and adjusting simultaneously the shape of the illuminated reflector from a sphere to a paraboloid, as shown in Figure1 (right panel).

Being the most sensitive radio telescope, FAST will offer astronomers chances to make great scientific discoveries, for example, neutral hydrogen surveys at distant galaxies out to high redshifts, looking for the first shining stars, detecting some thousands of new pulsars, exposing exotic objects, becoming the future host of the world (national, regional) VLBI network, etc. FAST will also help other researches like space weather studies and deep space mission services.
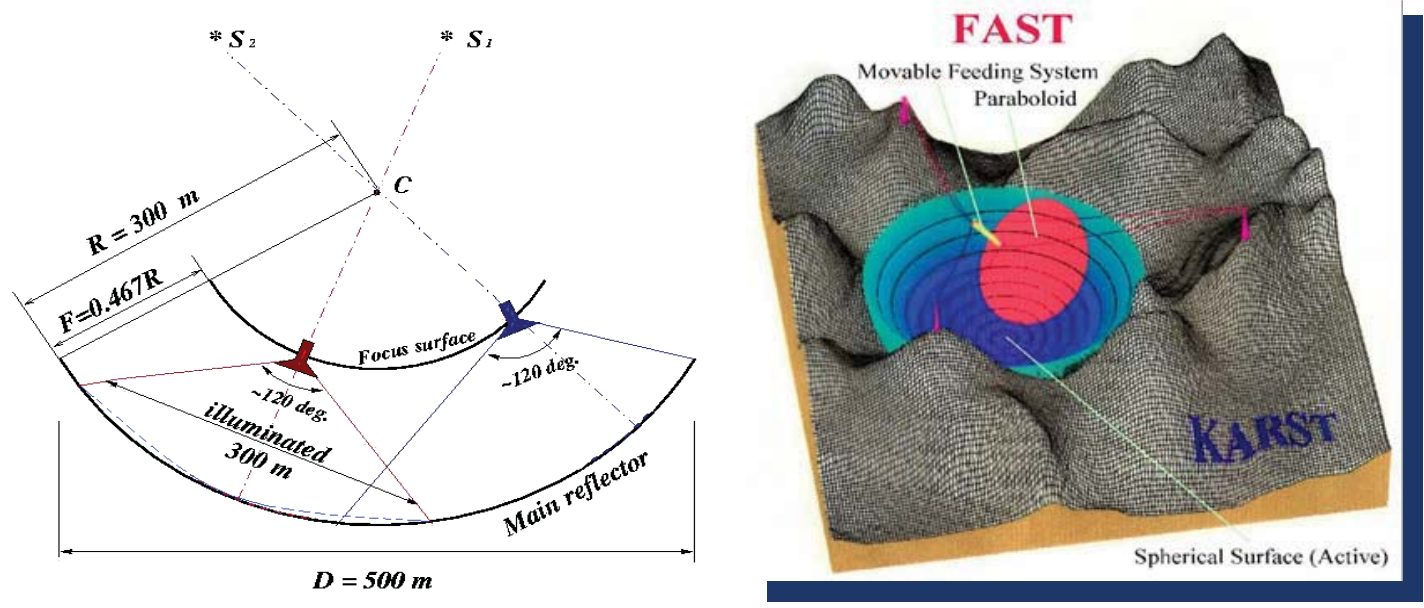

Fig.1 FAST geometrical configuration (left panel) and the concept (right panel) 
Progress on the five key technologies of the FAST project are summarized and updated briefly in this section, i.e., site preparation, main active reflector, feed support, measurement and control, and the receiver.

\subsection{Site preparation}

Guizhou province belongs to the subtropical humid monsoonal climate region. It is one of the provinces with better seismic stability, providing richest karst depressions, as potential sites for the FAST and Chinese SKA concept KARST. Comprehensive explorations on the site engineering and hydrological geologies, meteorology, electromagnetic environment, natural resources etc. have been carried out over the past 15 years. More than 90 earth sinkholes were mapped at high resolutions (for example, left panel in Figure 2), showing suitable profiles to locate large spherical reflectors.

A series of RFI (radio frequency interference) measurements were initiated with a portable equipment from 1994 at some potential SKA locations in Guizhou province, providing a snapshot of the situation in terms of spectrum, strength and temporal characteristics of the radio interfering signals, to check on the suitability from the point of view of RFI environment. The results showed that the radio environment was statistically stable. Since December 2003 an RFI measurement campaign had been conducted continuously for more than a year, to acquire data on background levels at potential SKA sites, by focusing on transmissions from directions around the horizon. Most of the signals can be attributed to known radio services. We conclude that, due to the remoteness of the candidate region and terrain shielding, the RFI situation in Guizhou looks promising, with little interference between $70 \mathrm{MHz}--22 \mathrm{GHz}$ (Peng et al., 2008)

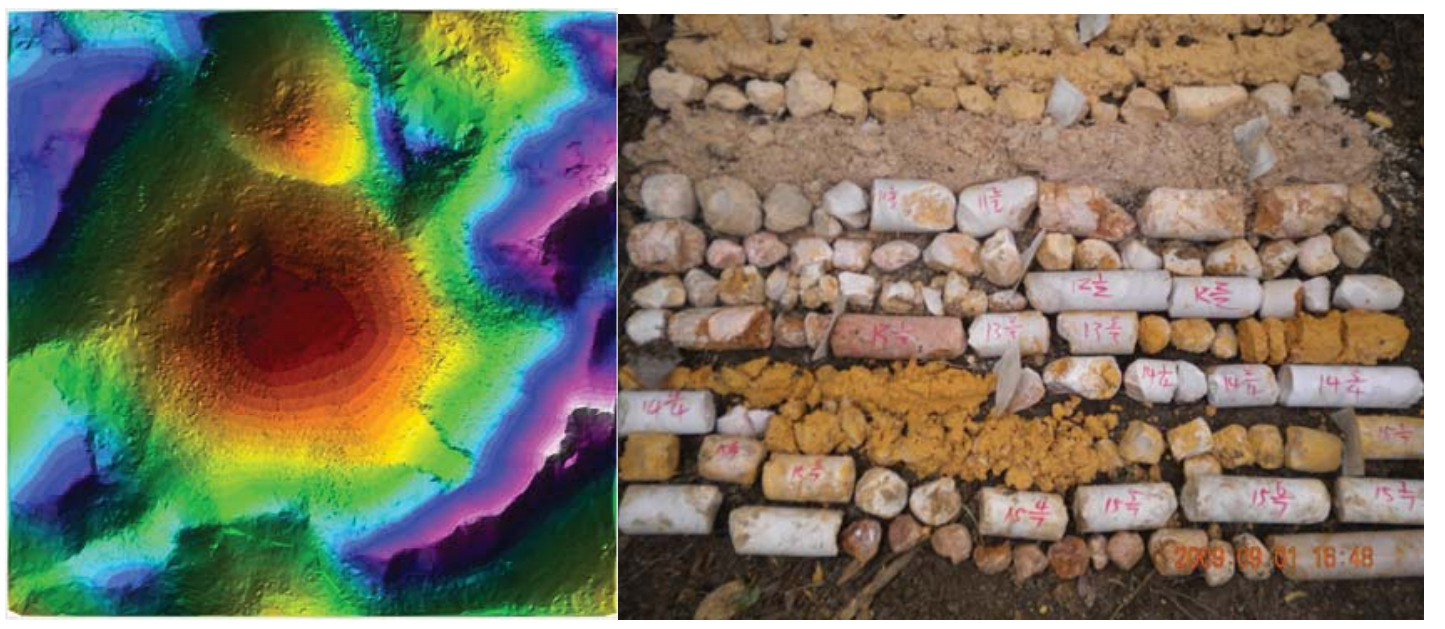

Fig.2 A high resolution map (left panel), and detailed site investigation by drilling experiments at Dawodang depression (right panel).

Site investigations by drilling experiments are presented in Figure 2 (right panel) at Dawodang karst depression, exploring the hydrogeology and engineering geology conditions underground. Dawodang depression at Pintang county, with its round shape and stable geology has been selected as the top one location for FAST, togther with its radio quiet environment.

The path of about $7 \mathrm{~km}$ long, as shown in Figure 3 (left panel), connecting the county road (at cross point NiuJiao) and the Dawodang depression, started construction around April 2010, 
and will be gone through by the end of the year 2010. Site accommodation near Dawodang depression for temporal use, in the period of constructing the FAST, is mostly completed, as shown in Figure 3 (right panel).

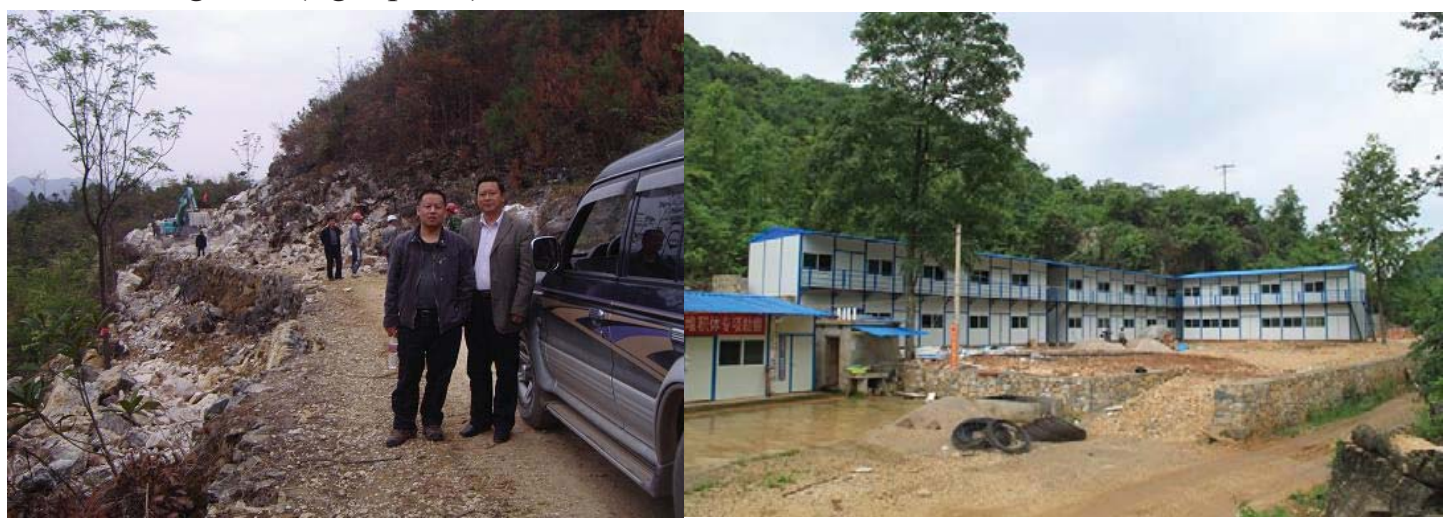

Fig.3 A path connecting the county road and Dawodang depression under construction (left panel), and site accommodation for temporal use built up (right panel).

\subsection{Active main reflector}

The illuminated part of the main spherical reflector (see Fig. 1) is to be active, which is continuously adjustable to fit a paraboloid of revolution by actuated control, synchronous with the motion of the antenna feeds in the focus cabin while tracking an object in the universe.
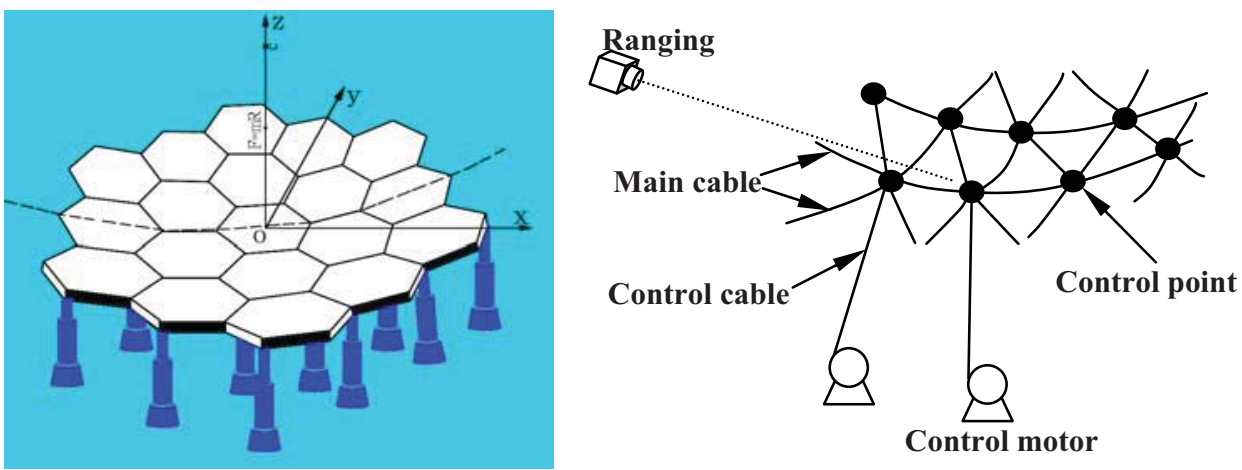

Fig.3 Reflector segmentation by hexagons with actuators (left panel), Cable-mesh spherical reflector (right panel): control points, cables and motors, equipped with additional ranging system.

To deform the main reflector, it is necessary to divide the giant spherical surface into smaller elementary units. Two different ways have been investigated to segment the huge reflector of the FAST: firstly, to have approximately 1800 hexagons. Each element has three actuators to point its position and connect it to adjacent elements, averagely speaking there is one actuator per element panel as shown in Figure 3 (the left panel, see Qiu, 1998). Secondly, considering that a reflector surface supported by a cable net could be activated to some extent without extra-servos controlling the lengths of the supporting cables, a similar structure to Arecibo telescope is adopted, a cable mesh consists of two sets of cables in orthogonal directions, as shown in Figure 3 (right panel, see Nan et al., 2003). A downward cable is used to realize control along the radial and tangential directions for a proper segmenting scheme, such as a 
Keiwitt geodesic triangle element panel shown in Figure 3 (right panel). Motors adjust these cables with feedback from the positioning system for the control nodes.

Both concepts have been demonstrated feasibly by intensive theoretical and experimental studies in 2001 and 2006 respectively. Compared to the first method, the cable mesh one is beneficial to construction of the FAST telescope in several respects: simplifies the structure of the FAST main reflector; eases machinery work and improves reliability; relaxes the fabrication accuracy and leaves space for further telescope upgrading; reduces the civil engineering between actuators and the ground. The advantages listed above may further be beneficial to FAST in terms of construction cost, time required for the project, operating reliability and telescope maintenance.

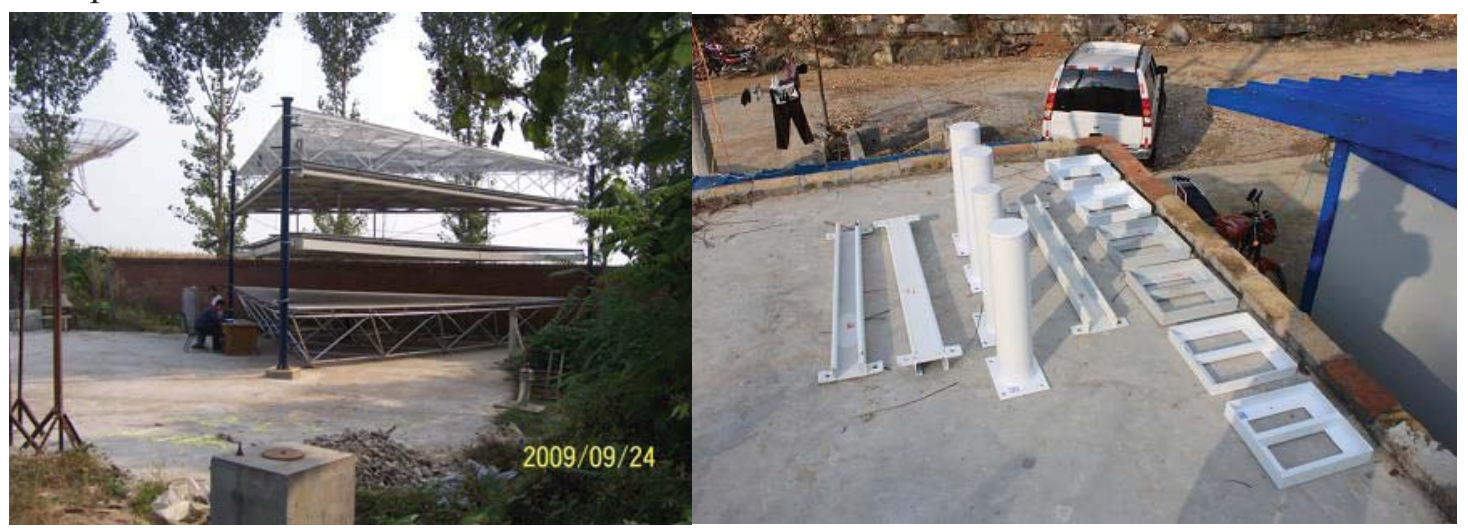

Fig.4 Some elementary reflector panels, in 1:1 scaled model, installed at Miyun Observatory in Beijing (left panel), and some key components of reflector tested at FAST site in Guizhou province (right panel).

Recently, elementary reflector units (left panel in Figure 4) in terms of different structure, technology and material, and actuators (left panel in Figure 5) to test strength, which are all in scales of 1:1 ratio, have been designed, manufactured and installed at Miyun Observatory in Beijing. Some key components, which are being tested for environmental anti-corrosion effects (right panel in Figure 4), and anchors to test earth strengths for anti-pullout (right bottom panel in Figure 5) were designed, manufactured and transported to FAST site in Guizhou. Wind environments were simulated with the map data near Dawodang area, as shown in the right top panel of Figure 5.

\subsection{Focus cabin positioning}

The FAST will be pointed by moving its focus cabin while the reflector surface is deformed. There are two research groups in Xidian and Tsinghua universities working at some different driving mechanisms, to study the feasibility of pointing a light focus.

A novel design for the feed-support structure of the FAST, as demonstrated in Figure 6 (left panel), was proposed by using six suspended cables, which is by means of integrated mechanical, electronic and optical technologies (Duan et al., 1996). There are three key components: first, six cables will be driven by servo-mechanisms with a central computer, secondly, a group of receivers will be mounted on a stabilizer in the focus cabin to provide a second adjustment. Thirdly, a laser ranging system will be adopted to measure the position of 
the feed in real time. The information will be fed back to the central computer for global control. To demonstrate such a design, a $5 \mathrm{~m}$ model was built first indoor successfully, preceded with a $50 \mathrm{~m}$ model outdoor in Xidian university, which has been under detailed development.
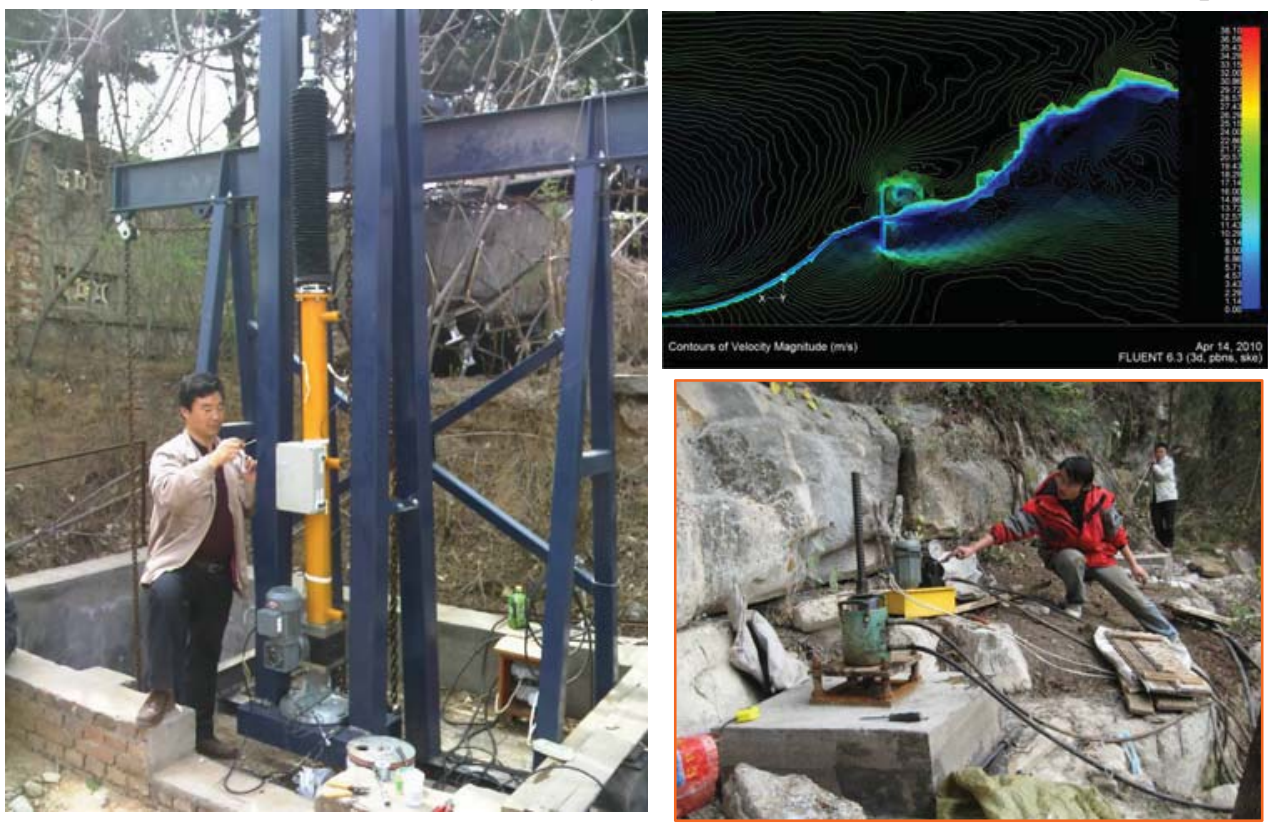

Fig.5 An actuator of 1:1 scale installed at Miyun Observatory in Beijing (left panel), an anchor coupled with downlink cable of the reflector tested at Dawodang depression in Guizhou province (right bottom panel), and a simulation result of the wind field environment of the FAST site (right top panel).
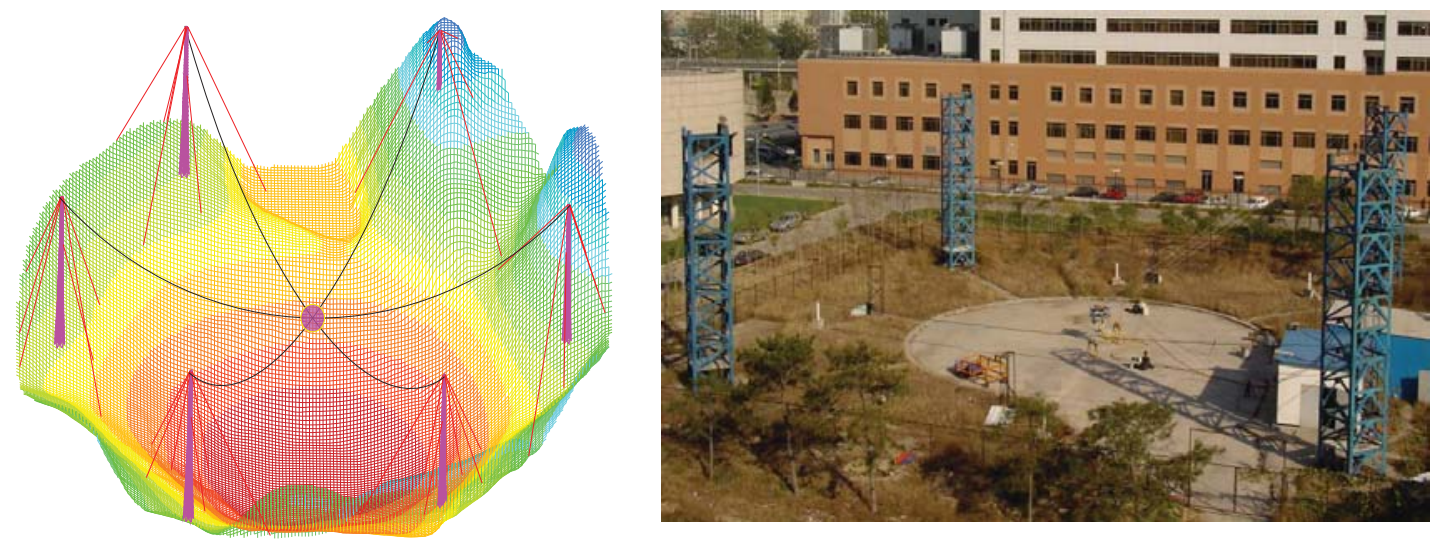

Fig.6 Feed support for the cable support without a platform: six suspended cables connected to mechanical servo-control systems with supporting towers (left panel); a $50 \mathrm{~m}$ model demonstrating the cable car configuration and pre-tension cables, positioning devices and controllers (right panel).

A similar but different approach was conducted by a group of colleagues in Tsinghua University. A small cable car, to house the focus cabin mounting feeds and receivers, is driven by two crossed pairs of parallel supporting cables, which are suspended from two pairs of opposite towers (Ren et al. 2001) as demonstrated in Figure 6 (right panel). This is more or less like a trolley on the cable-way in mountains. The pre-tension downtie cables are introduced to adjust the stiffness of the feed support structure. The main advantages of the cable-car 
configuration are: firstly, the maximum length of cable extension is relatively short. Secondly, downward cables could be used to adjust the stiffness and improve the dynamic characteristics of the cable support system. There were different scale models of 2, 5, 20 and $50 \mathrm{~m}$ dimensions established during the last decade, which are joint efforts by Tsinghua University and NAOC.

The end to end simulation for the feed support system was performed by a close collaboration with a German group firstly. Further development has been continued in order to contribute to the system design at NAOC, some prototyping experiments have been conducted at the top of the NAOC building in Beijing, at Miyun Observatory and Xidian University respectively. Recently, we have been designing the high towers, which heights would be above $100 \mathrm{~m}$ each, up to $\sim 170 \mathrm{~m}$ for the maximum one. It is desired to have the towers symmetrically distributed around the main reflector, while avoiding any risky earth work for their base foundations. Locations of the six towers have been optimized on the newly measured high resolution map of the Dawodang depression, further investigated and checked for the realization feasibility on site. In the mean time, we have been designing and selecting suitable optic fibres, which will be used for single transportation between the focus cabin, the top of the towers, and the ground laboratory. We have been testing fibre's lifetime while it bends. These efforts have been made closely together with professional industry people.

\subsection{Measurement and Control system}

The FAST is a telescope whose components are mostly movable. High accuracy measurements and control technologies are needed, to read the spatial coordinates of targets which will be controlled over long distance, while driving the focus cabin and shaping the main reflector simultaneously.

To point the focus cabin, two laser trackers of six degrees of freedom and four total stations would be employed, to observe the measuring targets mounted on the lower and upper plates of the stabilizer in the focus cabin, including one redundancy each for reliability and high accuracy insurance, as shown in the left panel of Figure 7.
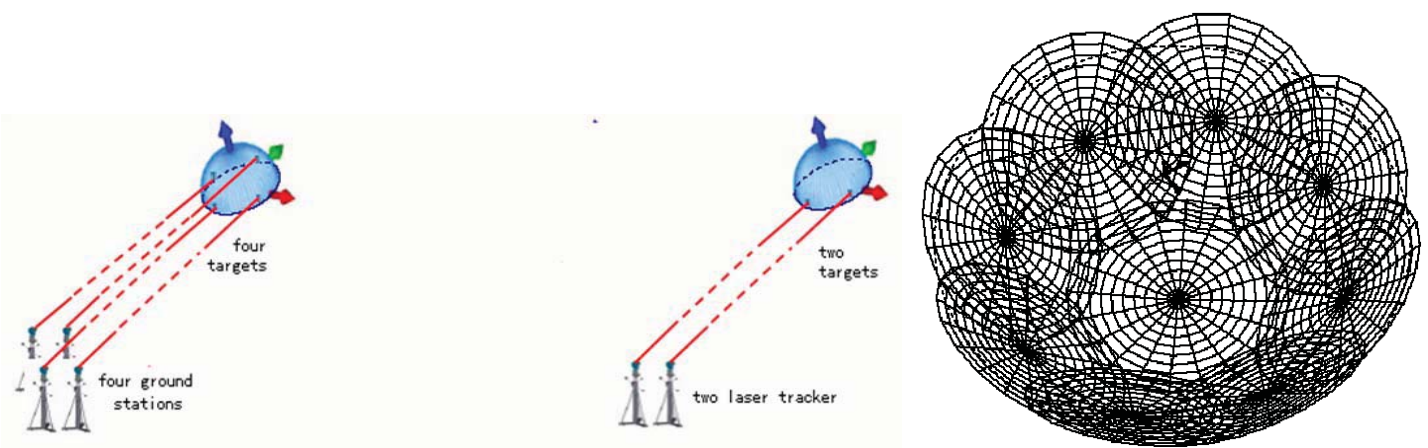

Fig. 7 Measuring instruments for driving the focus cabin (left panel): two laser trackers (right) and 4 total stations (left) will be employed; the shape of the reflector (nine sections) will be measured photographically by cameras (right panel).

To read the profile of the FAST active reflector, one should know the position of each node of the cable mesh. A photographic approach would be ideal to measure the surface, as adopted by the Arecibo telescope. Digital scanning instruments would be employed to measure 
the positions of the control points, which will be divided into nine overlapping circular sections, as shown in the right panel of Figure 7. Motors will adjust these cables with feedback from the ranging system for the control points.

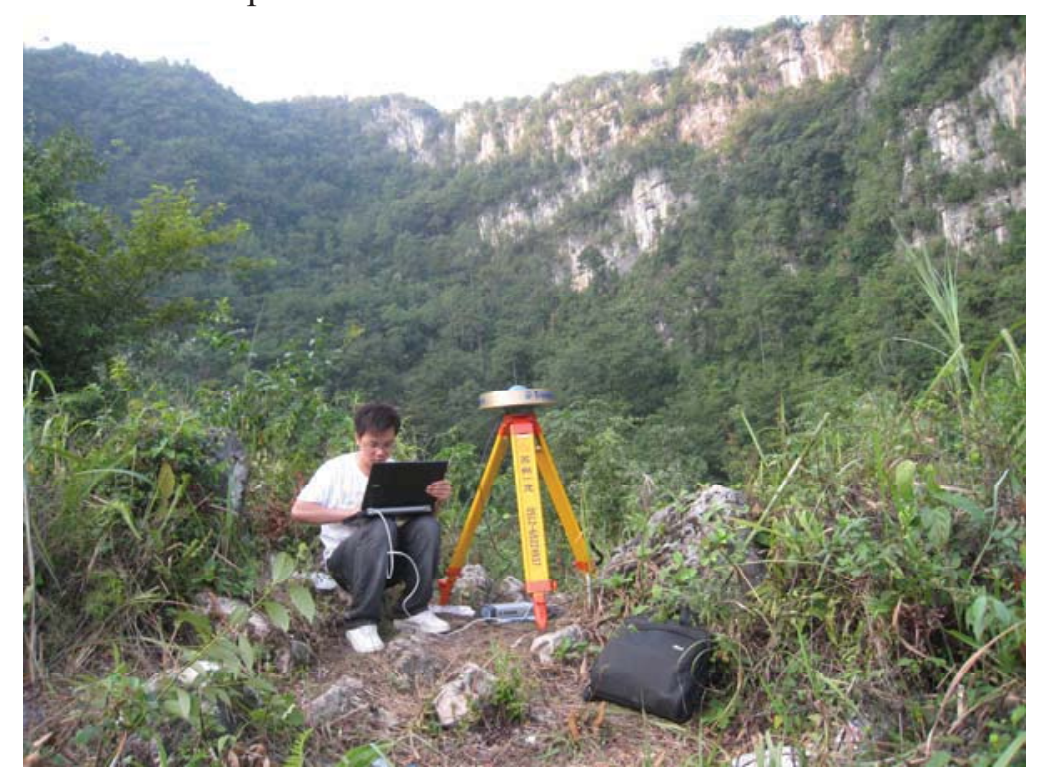

Fig. 8 Field observations with a GPS station were carried out at the FAST site in Guizhou.in 2010

The main reflector shape will be controlled in a semi-closed loop system, consisting of shape sensing and adjustment. A field bus technology would be applied to realize communications between the main control computer and controllers of winches. The system can also be supported by a flexible body control with additional information on the force of the cable. Locations of GPS (Global Position System) stations, laser tracker and camera supporters have been optimized on the newly measured high resolution map, of the Dawodang depression, and further investigated and checked for the realization feasibility on site. Some field observations with measuring equipment, like the GPS stations as displayed in Figure 8, have been carried out at FAST site.

\subsection{Receiver}

FAST receivers are to be mounted on a stabilized plate, connected to the main body of a hexapod in the focus cabin. The optics of the FAST shown in Figure 1 resembles a prime focus parabolic telescope with a maximum zenith angle of about 40 degrees.

A bilateral collaboration on the FAST project was established between the Beijing Astronomical Observatory (now NAOC, National Astronomical Observatories, CAS) and the Jodrell Bank Observatory. The joint discussion on low noise receivers is based on the use of existing, proven technology, to minimize the technical risk. A receiver layout was produced in 2001, followed by an update in 2006. The possibility of using the not fully developed focal plane array (FPA) technology was investigated either. The current layout uses 9 sets of receivers. A trilateral collaboration with CASS (CSIRO Astronomy Space Science) in Australia and JBCA (Jodrell Bank Center for Astrophysics) in United Kingdom was recently established to further 
optimize the receiver layout, and investigate the feasibility of an ambitious 19 beam receiver at L-band with $500 \mathrm{MHz}$ bandwidth, as shown in Figure 9. Optical fibers will be used to transfer the IF signals to the ground laboratory where the signal processing equipment will be located.

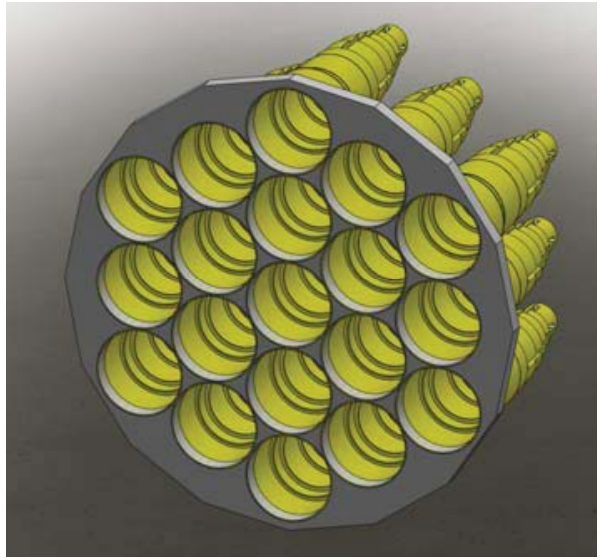

Fig. 9 A receiver layout drawing with 19 beams at L-band, investigated recently by a trilateral collaborations among NAOC, CASS and JBCA for the FAST.

\section{JLRAT for SKA}

The telescope FAST is now under final design, with preparation for construction around October 2010. It is expected to be available for astronomical use by 2015 .

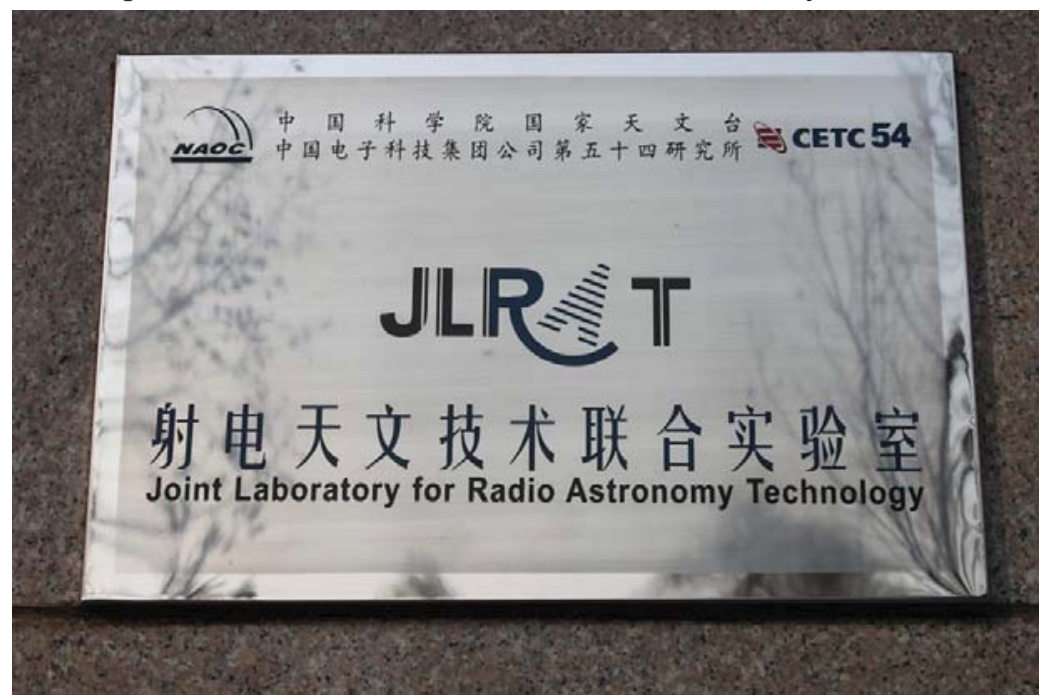

Fig. 10 A name board for the JLRAT between the NAOC and CETC54.

Considering frequent contacts for constructing the $50 \mathrm{~m}$ radio telescope in Beijing, the solar radio telescope array of $40 \times 4 \mathrm{~m}$ and $60 \times 2 \mathrm{~m}$ in Inner Mongolia, the $65 \mathrm{~m}$ radio telescope in Shanghai, and the ASKAP (Australian SKA pathfinder) antennas of $36 \times 12$ m, in June 6 2009, Director of the NAOC and President of the CETC54 singed a MoA to cooperate closely on joint development of the radio astronomy technologies. In January 12, 2010, Director of the NAOC and President of the CETC54 met at the opening ceremony to establish the JLRAT, Joint Laboratory for Radio Astronomy 
Technology, which is a joint effort to find more active ways for Chinese participations in the international SKA activities, like the DVA (Dish Verification Antenna) in the WP2 and similar approach in the TDP (Technology Development Project) in US; to develop a new mode to establish a radio technique laboratory; to serve better for future radio telescopes with better understanding each other; and to bring up new generation engineers for future astronomy service by making use of the existing resources.

The JLRAT will take the responsibility to coordinate the SKA activities in China, working together with industry companies, for Chinese current and future contributions to the SKA project in some ways.

\section{Remarks}

Before the FAST construction gets started, an office building in the campus, as a prepared phase for future Guizhou Radio Astronomical Observatory, is assigned to the FAST team by Guizhou University, which has been just decorated ready for use by the end of May 2010, as shown in the left panel of Figure 11.

As Prof. W. Gordon, who was the founder of the Arecibo radio telescope and the Observatory, recalled the sceptics, "We were young enough that we didn't know we couldn't do it", "If you dream, have big dreams, with supporters of talented people......", which was true for the successful construction and operation of the world largest single dish Arecibo $305 \mathrm{~m}$ telescope, which is true for the successful preparatory study of the FAST, and should be true for the successful construction of the FAST and the future SKA. The FAST dream is coming to be true with supporters of intelligent colleagues at its home Dawodang depression, to be shown up as that in the right panel of Figure 11.

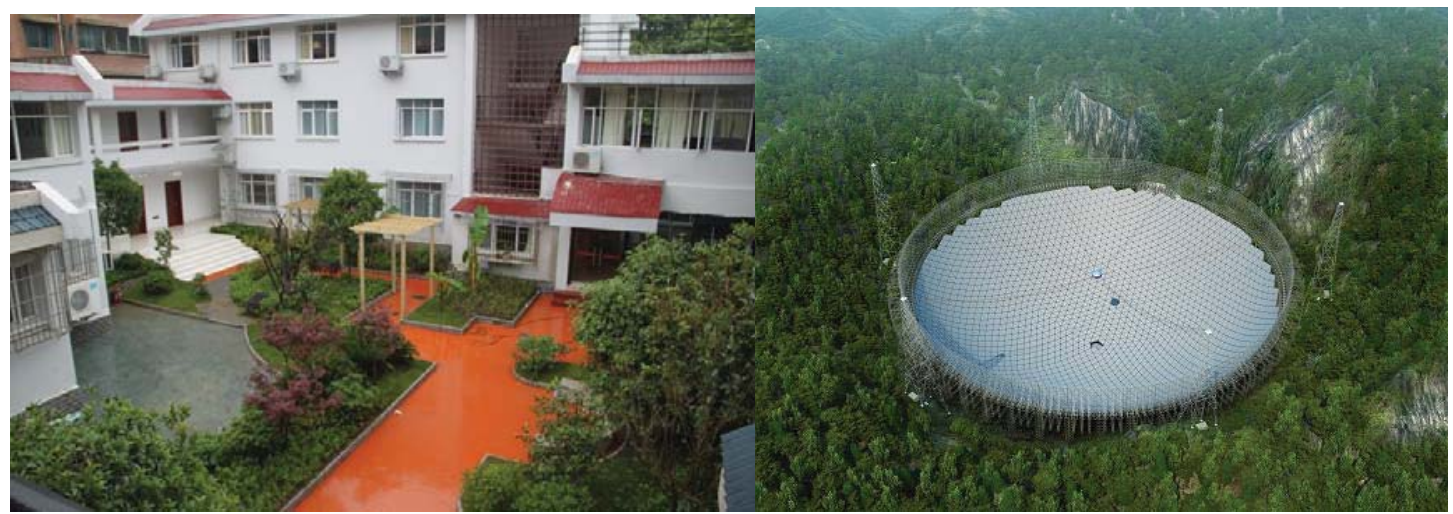

Fig.11 The office building yard prepared for Guizhou Radio Astronomical Observatory, decorated ready for use by the end of May 2010 (left panel); The FAST dream is coming to be true as collective contributions at its home Dawodang depression (right panel)

This paper is written up, based on the talk given by the author at the ISKAF2010, on behalf of the FAST project team. We would like to thank all the colleagues of the FAST/KARST project team for their contributions to the R\&D studies. We are grateful for financial support provided by the CAS, the Ministry of Science and Technology of China NKBRSF2003CB716703, and NSF Grant No. 10433020. Many thanks to Drs. Baoyan Duan, Yuhai Qiu, Yaoping Nie, Gexue Ren, Sebastian von Hoerner, Yukuan Mao, Jigun Xiong, Shengyin $\mathrm{Wu}$, Richard Strom et al., for their early great contributions to the big dream FAST. 


\section{References}

[1] B. Duan, Y. Zhao, J. Wang, G. Xu, 1996, Study of the feed system for a large radio telescope from the viewpoint of mechanical and structural engineering, in Proc. of the LTWG-3\&W-SRT, eds. Strom, Peng \& Nan, , International Academy Publishers. p 85

[2] R. Nan, B. Peng, Y. Qiu, G. Ren, S. Wu, Y. Zheng, W. Zhu, J.H. Wu, Y. Su, C.Jin, H. Zhang, L.Zhu, X. Xu, 2002, SKA Memo. 17

[3] R. Nan, G. Ren, W.Zhu, Y. Lu, 2003, Adaptive Cable-mesh Reflector for the FAST, ACTA Astronomica Sinica, v. 44 , Suppl., 13

[4] R. Nan, B. Peng, 2000, A chinese concept for the $1 \mathrm{~km}^{2}$ radio telescope, Acta Astronautica, 46 (12), 667

[5] B. Peng, R. Nan, 1998, IAUS. 179, Kluwer Academic Publishers, 93

[6] B. Peng, J.B. Li, T.Y. Piao, J. M. Sun, and R. Strom, 2008, Radio spectrum monitoring campaign at proposed SKA sites in China, PASP, 120, 625

[7] Y. Qiu, 1998, A novel design for a giant Arecibo-type Spherical radio telescope with an active main reflector, MNRAS, 301, p827.

[8] G. Ren, Q. Lu, Z. Zhou, 2001, On the cable car feed support configuration for FAST, Ap\&SS, 278 (1), 243

[9] R. Strom, B. Peng, R. Nan, 1996, Proc. of the LTWG-3 \& W-SRT, International Academy Publishers. 